\title{
The True Nature of Numbers is that they are a Group Associated with the Painlevé Property Mathematical life structures description hypothesis and notation
}

\author{
Yoshimi SHIMOKAWA \\ Kiiro Book Store LLC. \\ Tokyo, Japan
}

\begin{abstract}
The true nature of numbers is that they are a group associated with the moving Painlevé property. In the past, humans considered numbers to be individual entities. The twopoint selective ability of living beings can be considered to have established today's mathematical logic. We can consider mathematical logic to have been formed by the minimum condition of a consistent group (There is no discrepancies group) resulting from a light spectrum being transmitted to or reflected on a three-dimensional closed manifold. In other words, examining the characteristics of the light spectrum enables an understanding of the characteristics of human perception, and an understanding of the characteristics of the numbers perceived by human perception. Based on the understanding of these characteristics, humans think about perceiving mathematical logic. What was unfortunate in the past about the logical constructions that started with Gödel, is that they sought for the concept of numbers amid mathematical logic. Cellular automatons have advanced this approach by physicalizing it, and Conway revealed discrepancies by creating actual machines [12].The decimal system, in itself, is simply a notation tool and merely a notation. Owing to the structure of base conversion, it is convenient to use decimals in two-dimensional notations. However, decimals cannot be used in three-dimensional notations; instead, circular numbers are generated. This can be considered the result of using " 0 ," although the concept of writing out a three-dimensional notation consistently should be created separately. With no concept of null $(\boldsymbol{N})$ in the "Set theory and the continuum hypothesis"[5], that the perception of the human body is simply maximizing the margin of classification boundaries in a SVM problem [3]. How to consider maximizing the margin of classification boundaries in perceiving the problem could be thought of as the theme for the theory of numbers. Current, by "The International System of Units (SI)" has been defined, A unit got association that the unified. By using the unit, it will be elucidated also algorithms of the molecular structure in the organism. We need a third algorithm. It is the algorithm of the molecular structure organism, that is"the number of recognition" and "the number of physical"Is an algorithm to unify. In other words, temporary has been described structure in this paper, a common algorithm that is "the number of physical" and"the number of recognition". Once again, it was discussed the number of nature, for that algorithm. In the future, to make a key operation using this algorithm. I wish to apply to display notation the development of new devices.
\end{abstract}

Keywords-cognitive science; pattern recognition; scale invariance; Painlevé Property

\section{INTRODUCTION}

\section{Preconditions and Assumptions}

If we were to assume that the two-point selective ability has established the present day mathematical logic (the foundation for classification boundaries), it is necessary to understand its characteristics. First, I considered Conway's "puffer train" as a two-point classification boundary figure structure. It has a main body portion that moves independently at half the "speed of light" without interference from fragments, and two "spaceships" that attain two-point discrimination. For considering a nontrivial self-renewal process, a binary method with two-point discrimination provides the required confidence level. When introducing the physical settings on a computer, this is the resulting appearance because the notations on the display are limited to two dimensions.

Next, It is considered why organisms form clod-like continuous shapes from chaotic locations. It is considered examples (of such growth) in nature as well as of organisms that grow in the above mentioned way in nature. Then focused on portions of organisms that remain motionless as objects instead of as growth, on the opposite axis of discrimination. Through the induction of epitope light, plants ultimately produce fruits that can be counted-one, two, three, and so on. Fruits are considered complete after separation from its main body. In other words, the perception of "separation" in the boundary problem is thought of for organisms as being conducted by epitope induction, some other condition (algorithms) for displays. Then examined the boundary problem for this object formation and numbers, and then created 3D figures illustrating the method by which those shapes can be transformed from "Concept 1," which is explained below. By using a three-dimensional multiconnected closed manifold, "the difficulty of classifications" is interpreted along with the state of changes.

\section{Question the continuum hypothesis initial setting}

The claim of this research questions the initial settings of the continuum hypothesis. My claim is that numbers are "a simple concept arising from the human awareness of twopoint discrimination, and its symmetrical transformation patterns" and that the concepts of the natural numbers 1, 2, 3, $4,5,6,7,8$, and 9 are an expression of how this two-point 
discrimination is moved. To avoid confusion, the concepts of natural numbers will be referred to below as "Concept 1," "Concept 2," "Concept 3," and so on. Cantor and Gödel focused on cardinality as well as ordinal numbers, but my approach goes a step further, taking the view that cardinality is perceived by the human body, which is created by antigenic epitope induction.

\section{Scope of Analysis and Method}

The analysis covers three areas.

1) Organism model analysis

2) Mechanical model analysis

3) Conceptual model analysis

In other words

1) classification boundary epitope induction

2) classification boundary algorithm

3) classification boundary cognition

In other words

\section{1) Analysis of the organism exponentiation conditions save deformation}

2) Analysis of the ends of the puffer train and the two spaceships described in The Recursive Universe

3) Analysis of tori (three-dimensional multi-connected closed manifolds) that represent the transformation of Concept 1

To analyze the binary numerical machine minimum model for Conway's puffer train described in The Recursive Universe (Item 2)[12], Be inferred the property of life (in other words, the independence that is characteristic of life), from a different perspective by making a comparison to the conditions of a closed manifold. To analyze the organism model (Item 1), Be inferred numerical formulas from epitope induction, examining "copying" numbers and "control or stop" numbers that can be linked to the binary system. In other words, cell division was interpreted as light induction using copying, and the transformations of Concept 1 (Item 3) were analyzed from the state of perception in which understanding changes when position changes. In other words, the manifold of Concept 3 was displayed.

The minimum figure of perception for considering the logic of numbers is the binary machine minimum model described in The Recursive Universe (Item 2) as the logical theoretical framework. It is considered co-dimensional problems that generate discrepancies in mathematical logic to be inseparable from the structure of perception. It analyzed the structure of the location of visual perception using an organism model, and viewed it along with its constituent equations. Then surmised other examples of this kind of equation being used for other organisms. Then, cognitive recognizes the "concept 1", also, how to recognize a mistake as "concept 2" and "concept 3", I shown in the figure. It was constructed a simple figure with the whether $3 \mathrm{D}$ can be deformed in any way from the "Concept 1". Using this 3D multi-linked closed manifold, changes were observed regarding "The difficulty of classification" at the same time.
Comparative analysis targets three, respectively

1) physical status quo analysis

2) physical state compression model analysis

3) cognitive status analysis That is the meaning.

\section{ORGANISM MODEL ANALYSIS}

\section{A. Number of Visual Perception Iris Structures}

First, I used mathematical logic to examine the two-point discrimination structure of two eyeballs. It is considered discrimination to be the margin maximization of a classification boundary in an SVM, and searched for its discrepancies. Vision (i.e., light-gathering) done by organic perception is structured with the structure of the iris (the higher-order equations for " 3 ," "5," and "6"). However, when viewed through a vector analysis of a three-dimensional space, this structure resembles a Seifert surface structure with topology classification E6. It is a 52-dimensional real Lie group composed of four automorphic mappings over four rings. It is a space given by a closed curve $\mathrm{C}$, meaning that it can be created without $S$ and $L$ touching. This is the base of perceptual cardinality. Seifert surfaces are composed of areas with four boundaries and linking numbers. From there, strokes are added to two locations and by folding over to the inner side, three rings overlap on the inside[7]. This is a knot structure called a (p, q)-torus knot, and in two dimensions, it is also a $3+1$ threshold discrimination structure. Spaces that resemble the horizontal figure eight in two dimensions are two-threshold discriminations, but they can be made consistent (no contact between discrimination spaces $\mathrm{S}$ and L) when they have a butterfly stroke structure in three dimensions. In other words, they can be considered a 1threshold discrimination in three dimensions. This space is a simply connected 3-manifold floating in a temporary 3sheeted covering space at the center of a simply connected 3 dimensional closed manifold. By using these four homeomorphisms, adjustments are perceived, and far-point perceptions can be surmised.

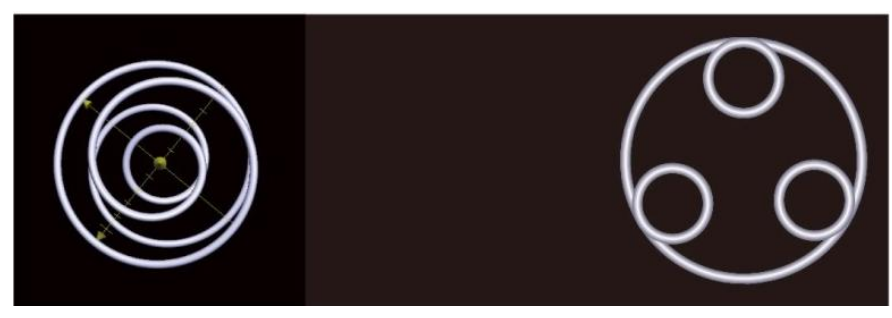

Fig. 1. Seifert space, in four of areas, have been constructed. Even when it is overlapped with the complexity, even when just break up into three regions and one of the areas, Linking number of them does not change

From the opposite perspective, even for the same twopoint discrimination, a two-point discrimination of an included area is structured from the overlap of three portions. In other words, vision is defined in terms of a simply connected 3manifold and two-object discrimination, but these can be considered as an E3 multi connected three-dimensional closed manifold. Doing so secures a spot in only one region, enabling a stable and consistent discrimination in the interior. 
Organisms perceive the world using the "simply connected three-dimensional closed manifold in a temporary 3-sheeted covering space" as the source of reflection. In other words, it is possible that conditions omitted from this intricate structure are not perceived. To put it differently, the perception used by organisms is one for which this structure is consistent.

From the discussion above, the human visual perception can be considered to be able to discriminate 9 regions (3 points, 3 locations) using the continuum of concentrated light.

\section{B. Number of Epitope Inductions Composing Plants and Animals}

Strictly speaking, a two-point discrimination can be considered two special locations in a three-point discrimination of a convex function. Although there is an origin serving as a harness that moves the two locations, in the case of the structure of the spectrum and iris, it indicates the light-gathering act from the outside.

Pursuing this discrepancy reveals the cardinality of numbers and the discrepant portion of the decimal system. It is considered the origin of perception by organisms (which begins with the two-point discrimination) to be Concept 2 and Concept 3 , and the final stage of perception by organisms to be Concept 5 . The concept currently called " 3 " can be broken down into " $2+1$," with the " 1 " portion of " $2+1$ " being an adhering item. In other words, It is considered generating Concept 4 to have a common harness. Furthermore, Concept 3 has self-homeomorphism, and Concept 6 is the result of moving unchanged to a higher order and becoming threedimensional. It is considered the $(n+1)$ pattern that moves to a higher level and has an external origin acting as a harness to be Concept 7 . If considered in terms of the algebraic method, it is what becomes the characteristic function.

Among the Painlevé equations, the third-order equations are the same as the equations representing the structure of the iris, which is the source of visual information. These Painlevé property have four singularities: a moving pole, infinity point, point 0 , and point 1 . Among these four singularities, the moving pole is the source of the relativity theory and the infinity point is the source of the theory of cosmological inflation. The shape and characteristics of the universe are explained using the characteristics of light. For the dispersion point/separation point directly before point $0 /$ point 1 , on the other hand, Dr. Barbara McClintock used the characteristics of light to explain the shapes and characteristics of terrestrial plants [1]. In terms of separation/dispersion, the location affected by the real array is the antigenic determinant (epitope). In other words, the iris perceives both the far point and the dispersion/separation point.

When explained in terms of a garlic that is a pentagon and okra, antigenic determinants that react to light correspond structurally to the origin of the Hasse diagram, and can be considered radicals that manipulate genetic information through the action of light. In terms of signal logic, they correspond to a harness. The types with two axes passing through them may be describable by second-order equations, and types with three axes by third-order equations.
Now It will discuss the shapes of plants. A normal garlic bulb (Photo 1-1) is formed by a Concept 5 epitope, while a garlic bulb with a shifted focal point (Photo 1-2) is formed by a "5 + 9" epitope. An okra pod is formed in an inverted manner by an epitope of Concept 5, but the internal structure of the pod is formed by a seed using 14 epitopes in the inverted cavity. The cross-section of a normal garlic bulb (Photo 1-3) has a mathematical logic in which a complexplane curve circles while spinning toward the center, with the starting and ending points aligning. In a garlic bulb with a shifted focal point, the epitope formation of Concept 5 becomes shifted such that the end point of the first "lap" does not overlap, causing the $\mathrm{H}_{2} \mathrm{O}$ ions (a constituent of the formation) to reverse its polarity and make two "laps" with "5 $\times 2$ " -1 . In other words, Concept 1 results in this case because Concept 5 of a normal garlic bulb (which is the "one before" in a quinary calculation) is interpreted as Concept 1 in the epitope. The second "lap" of the shape formation can be considered a covering space in the numerical calculation.

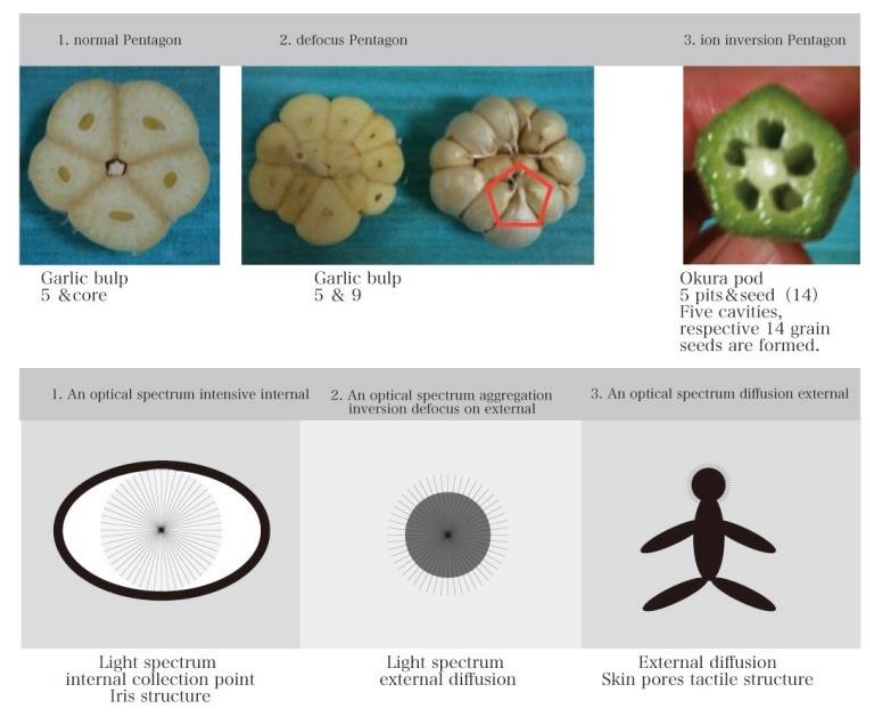

photo1 : 1. normal Pentagon 2. defocus Pentagon 3. ion inversion Pentagon

Fig. 2. Of visual and tactile, perception condensing reversal

Each of the pores along the human skin has individual epitopes, and is sealed by pentagon-shaped molecules. From there, two "tails" extend from each pore, leading to the foliation structures under the skin. For the eyeballs previously mentioned, visual acuity is determined by two-point discrimination with two eyeballs, but the discrimination with human skin may differ according to the day/night lighting and temperature differences. In other words, temperature differences may be used for the detection of the maximization of the margin of classification boundaries in an SVM problem with cardinality[3]. The light structure from the spherical surface used by vision can be considered a Gaussian mapping on the skin in the case of touch, and used in touch perception. Skin cells are replaced in 28-day cycles (peaking after 14 days). Since the human body is also an aggregate formed using the epitopes of Concept 5, it can be inferred that the skin surface (sealed with the epitopes of Concept 5) uses the same identification number as okra (Concept 14) for replacements. 


\section{Reaction-diffusion Path Estimation and Number of Equations}

The panda's coat of fur is a special example of predictable transmission. The panda has a diet of bamboo grass with strengthened epitope paths, and has a coat of fur identified by a distinctive two-color pattern. Juvenile pandas that do not eat bamboo grass do not have the distinctive two-color identification pattern. Among humans, the birthmark known as the Mongolian spot that is prevalent among those of Mongolian descent may be due to an accumulation of B12 cobalamin (which contains a cobalt molecule at its center) at the final portion during the final stage of ecological formation from epitope transmission. The birthmark disappears when the half-life of cobalt ( 3 to 5 years) has elapsed. The mathematical model of the transmission path of these epitopes simulates a signal network of a group of cells.Pattern of these plants and animals are thought to possible written in mock function, which is a Exponentiation conditions save deformation.

Some examples of epitope inductions with reversed ions are as follows. Among plants, there is the example of lotus petals, which are formed as 16 " $22^{22 "}$ petals with the third-order equation " $\mathrm{n}^{\mathrm{n} n}$." Epitope induction No. 17 mays occur in the hollow stalk created from negative ions. When the shape of the lotus root is " $\mathrm{n}^{\mathrm{n}-1}$ " " $3^{3-1}$ ", it is created by the second-order equation for "9," which may be because the polarity of the ions in the underwater portion of the lotus root is reversed. Another example is the second molar tooth, which has the same structure as the lotus. The visible portion is composed of four mountain shapes, and the ion-inverted root portion has three prongs. The first-order equation $(n+1)$ that represents Concept 17, can be considered one of the convergence epitopes in the human body. Object programs that represent computer units (binary) can all be the result of the epitopes having a heteroclitic-loop reaction-diffusion path.

\section{COMPUTER DisPlay ANALYSIS: NUMBERS OF ENDS OF THE PUFFER TRAIN AND TWO SPACESHIPS}

The ends of the puffer train and the two spaceships described in The Recursive Universe are paired with each other, and are related in three dimensions like points of contact in a Seifert surface. A non-cyclical region with a pulsar and the leading edge of the train are at the top and bottom of both edges, respectively. This arrangement can be considered to be merely the result of planarization of the left/right orientation of molecular structures existing in higherdimensional worlds, which itself is a polytope of compressed notation.

Ultimately, it is the moving objects that are "fixed," and the puffer train has a bottom and front. In other words, the puffer train has directionality, and the existence of this directionality is considered the true nature of movement. Having directionality implies that symmetry is spontaneously broken. This spontaneous breaking of symmetry is the "movement" that gives a region its independence from the background, or what could be considered "life-like."
The relationship between the main body portion that moves independently at half the speed of light without interference from fragments and the two spaceships that attain two-point discrimination can be expressed by a tau-function relationship represented by three objects aligned in a straight line. When considered as points in a 5-dimensional projected space, they become consistent. The packaged structure is seen in molecules such as collagen. When considered only in terms of mathematical logic, it is the result of a triangular pyramid calculation formula consistently describing selfhomeomorphism. For the six tau functions[9], the three dependent variables of the symmetrical forms correspond to the three $\mathrm{f}$ variables.

In other words, the fixation of objects is a temporary fastening supported by directionality in at least two directions. It is balance that appears to be stopped, and this is the true nature of "stoppage." To put it differently, the true nature of a three-dimensional closed manifold is supported by equations of at least the third order, and it is a balanced, closed object bearing the potential for movement. The appearance varies according to the dimensions in existence, and it is the portion thought to have a covering space in quantum physics. It is considered interpret this fixed balance as Concept 1.

Both ends of Conway's puffer train and the two spaceships described in The Recursive Universe are a pseudo-open space with a barrier and are closed by two dimensions. However, by making their length massive, they become a simulation of infinity. What this signifies is a pseudo-open model enclosed by straight lines, but this could be a length calculated as a curve. This could also be a pseudo-model for space that humans once perceived to be opening or continually expanding.

New solutions can also be obtained for polyhedral objects by using curves on the surfaces of the Goldberg solid body. The higher-order equations " 3 ," " 5 ," and " 6 " are used for these solid bodies. When these facts are linked objectively, the logical shapes that form nature can be considered to be dependent on curves. The recognition that straight lines are special cases of curves may further advance the potential of science. " 3 " can be considered the minimum figure of selfhomeomorphism, " 5 " the minimum figure of the condition of stopping with bridging [8][2], and " 6 " the minimum figure of dimension-raising using two self-homeomorphisms. Conway created a two-dimensional model of "life" that moves autonomously and has self-homeomorphism. In other words, it can be said that the figure was created which becomes the fixed balance of Concept 1 that is independent from outer space.

However, the Seifert structure of the eyeball represents a 52-dimensional real Lie group E6 (composed of four geometric automorphic mappings over four rings) where it is built infinitely externalized internally [4] [6]. In other words, it can logically be considered a structure able to perceive a faraway point arbitrarily close to a straight line. 


\section{ANALYSIS OF TORI (THREE-DiMENSIONAL MUlTI CONNECTED CLOSED MANIFOLDS) THAT REPRESENT THE TRANSFORMATION OF CONCEPT 1}

\section{A. Perception Analysis of Concept 1 to Concept 9 Concept 1}

signifies a simply connected three-dimensional closed manifold and a multi connected three-dimensional closed manifold.

\section{Concept 2}

signifies the action of maximizing the margin of classification boundaries of two-point discrimination.

\section{Concept 3}

signifies the minimum number of automorphic constituents; a polytope towards two dimensions.

\section{Concept 4}

signifies center 0 at the time of nonlocal stable opening/closing.

\section{Concept 5}

signifies a simply connected higher-dimensional open manifold; a polytope towards three dimensions. For thirdorder Painlevé equations, " 1 " and " 5 " are processed as the same simple group, where "R5/2" and "R $1 / 2$ " contained in a sphere of radius $\mathrm{R}$ are the same covering space of a cyclic group.

\section{Concept 6}

signifies $2 \mathrm{n}+0$; a diploid of a perfect number with the minimum self-homeomorphism in three dimensions (Concept $3)$.

\section{Concept 7 \\ signifies $2 \mathrm{n}+1$.}

\section{Concept 8}

signifies $\mathrm{n}^{3}+0$; " 4 " in three dimensions, along with its covering space and origin 0 .

\section{Concept 9}

signifies $\mathrm{n}^{\mathrm{n}-1}+0$; " $\mathrm{n}$ " in three dimensions, along with its covering space and external origin 0 .

The transformation of Concept 1 is represented by tori (three-dimensional multi connected closed manifolds).

The compact theory (Kaluza-Klein Theory) of the codimensional portion that encloses the independent Concept 1 described contains a discrepancy [11]. The reason for this discrepancy is a question of how the locations interpreted were changed and the interpretation of locations is related to the problem of perception. Accordingly, It is considered in terms of the number of material structures that support perception as well as the sense of sight and touch, and the number of epitope inductions using characteristics of light that are indispensable for perceiving organisms. When reexamining what numbers are in terms of these locations covering spaces, It is considered the problem along with its initial settings-living things that perceive numbers. Human perception, in particular, is composed of what resembles a covering space of higher-order equations. The homeomorphism of the mathematical structure of the iris may be reflected in the target and create visual perception.

From the observations above, the true nature of numbers can be considered a creation of the rationality that describes "life." In macro terms, everything can be understood from the shining or reflection of the light spectrum-from the molecular structure of minerals to the trajectories of celestial objects.

Humans have moved from the age of paper and writing instruments used to make compressed notations in two dimensions, to the age of recording transmitted manuscripts enabling uncompressed notation. The age of notation using light has begun. Writing on the two dimensions of paper is a notation method that requires the compression of codimensions, and the decimal system is designed mainly to handle this application. Protists are completed in five decimal world,However, achievement that won the longitudinal and movable creatures, Have adopted the decimal system, which was to have a symmetry in quinary. However, notations that do not compress co-dimensions are needed when drawing non-orientable organisms such as sea urchins, oriented manmade structures, the shifting of orientations that occurs in outer space, or the structure of viruses. While computational models already exist, there are no comprehensive concepts or operation keys. Therefore, new connotations for these new notations are required. As the mathematical logic and threedimensional computations are both ready, keys used to write extra dimensions are required for use as "procedure words."When re-examining the nature of numbers in terms of these types of locations (involving folding co-dimension) covering spaces, I considered the initial settings-living things that perceive numbers. The only reason for doing so was that the locations in which number discrepancies appear are special intermediate-dimensional planes (i.e., co-dimensions), where the locations in which the settings of words, perception, and tools are vague or poorly set. My discussion has been advanced by using "procedure words" instead of "sequences/quantities" as the words of mathematics, defining perception as "two-point discrimination" and using the decimal system as the current tool of notation.

I have derived the problem of the divided perception of a set known in a group from the structure of human perception, which is based on two-point discrimination, by approaching the problem using a model involving simple movements in mathematical logic.

\section{DISCUSSION}

\section{A. Control Numbers}

Control numbers are locations having extra dimensions, in which the characteristics of light are noticeable, or locations, where shifts can be created with their reflection angles. " 5 " is a condition that consistently (There is no discrepancies) closes a boundary as a co-dimensional polytope in a regular polyhedron in three dimensions. Since, in general, it is not 
possible to solve fifth-order or higher algebraic equations by the Exponentiation root, fixed form transformations using Exponentiation symmetry are not possible. In other words, this means that the path for epitope induction is not definite. Even in the evolved organism of the human body, the fingers, toes, and body as a whole constitute objects that have stopped by dividing into five branches. In other words, the creation of "undividability" can be considered a condition for controlling or stopping convergence. Therefore, Concept 5 is the number that functions as a controller. If observing based on the characteristics of an individual, primitive organism such as sea urchins are independent individuals that have their entire structure formed by a simple equation for "5." Sea urchins have been shaped to withstand the conditions of the highly acidic primitive sea, (in other words, conditions where the state of ions are different that of today) and can be considered to have a shape that is a reaction to the numbers at the rear. The acidic sea can be considered the neighborhood(affect around, and distributed the separation point) condition for creating organisms with acute-angle shapes. In terms of numerical formulas, these organisms may be patterns of manifolds with the origin of epitope inductions (which mammals have inside their bodies) that moved to the outside and have been induced by the 17 types of metals (halogen metals) present in the composition of the sea.

\section{B. Number of Inductions}

Contrary to the control numbers, morphogenesis up to immediately prior to the five-dimensional stoppage number depends on the "substitution group." As can be seen from the example of the lotus discussed above, even in a case where the appearance expresses such numbers as "9" for the hollow stalk, with " 16 " petals and "1" lotus Exponentiation for the flower, it may possess conformity in the group overall simply by virtue of the fact that the root to be taken differs due to ion inversion and swinging back by the substitution group operation. This can be rephrased as it being engaged in monodromy preserving deformation. In other words, the number of inductions is equipped with a Painlevé Property.

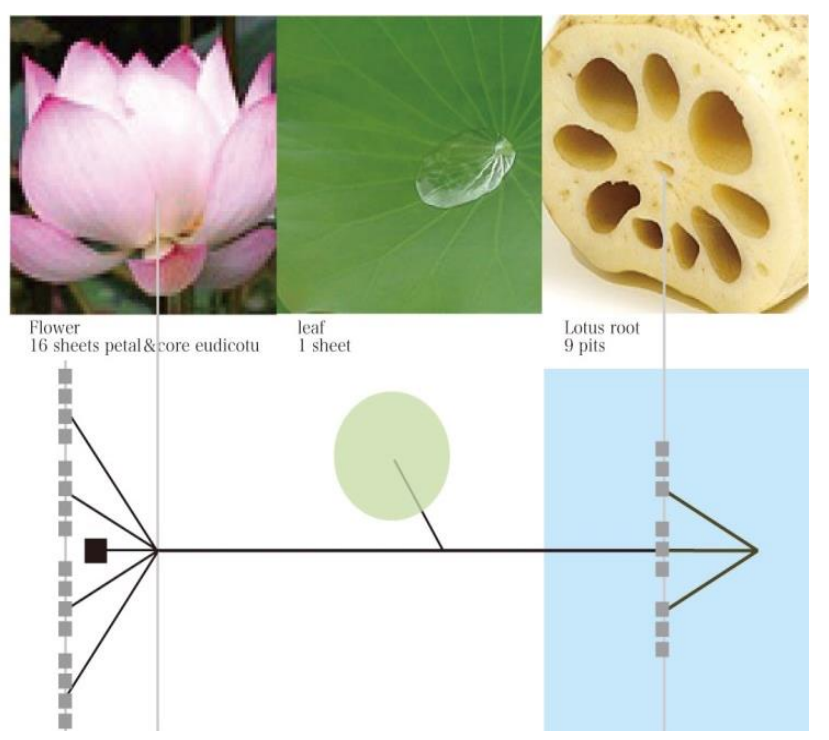

Fig. 3. Ion, plus or minus reversal and transformation It was on the basis of the Euler pentagonal theorem
Moreover, if one considers a case in which animals and plants are established as solids to be "Concept 1," this includes an agglomeration (DiscretelySubset)of the discrete parts of number $\mathrm{R}$.

Epitope induction generally consists of two-part division and three-part composition, and is thought to move in accordance with the knot theory. Induction lines that can be calculated using Gröbner bases are thought to exist on the knots or crossings. The number of twists is thought to be related to speed.

\section{Perception of Numbers}

Initially, the idea for this research arose from wondering if an attempt could be made to prove non-independence using the continuum hypothesis. As my hypothesis, It is considered what the result would be if the perceived numbers " 1 ," " 2 ," and " 3 " were not independent - in other words, did not have the characteristic of being a sequence. The answer is chaos. However, when It is considered how " 1 ," "2," and " 3 " could be created as concepts when considering chaos as a single set, I also considered the case of perceiving Concept 1 as Concept 2 in a three-dimensional space, and Concept 2 as Concept 3. As pursued these facts, It noticed that when the concept human perceive as Concept 1 takes on a different appearance, it becomes Concept 2 and then Concept 3. Fig.4 shows the transformation and regeneration of a doughnut-shaped torus of a three-dimensional open manifold normally perceived as " 1 ." Concept 5 viewed from above in three dimensions can appear as " 3 " or " 2 " when the representation is compressed into two dimension and viewed from the side. Ultimately, it can be transformed into a state enabling the calculation of its homeomorphism to a simply connected three-dimensional closed manifold. While the numbers discussed here are mere concepts, in practice, they can also be considered characteristics of the light spectrum composing living things.

It is necessary to establish boundary classifications and domain theory that use two-point discrimination cognition, the basis for classification boundaries, and that are in line with reality. It is proper to think of boundary classification as pattern recognition of an n-dimension polytype. The way of approaching the question in the continuum hypothesis was simply regrettable, and it is interesting as training in the method for thinking. Adopting Dr. J H. Conway's expressions of haploid and diploid for doing structural pattern recognition for the cardinality of the number are thought be in line with reality and appropriate[3][4]. This is because structural pattern recognition uses a Hasse diagram and preserves the consistency as a group with an orientation. To phrase it differently, it serves as a method for coming and going through an intermediate dimension consistently(There is no discrepancies ) .It is considered as characteristic of the optical spectrum.

\section{CONCLUSION}

\section{A. The number of coming and going Topology}

Organisms may use these characteristics to construct organic structures. For example, the external appearance of an individual tangerine can be viewed as a simply connected 
three-dimensional closed manifold, but the internal structure can be considered a transformation of a doughnut-shaped torus of a simply connected three-dimensional closed manifold. The same applies to bulbs of garlic.

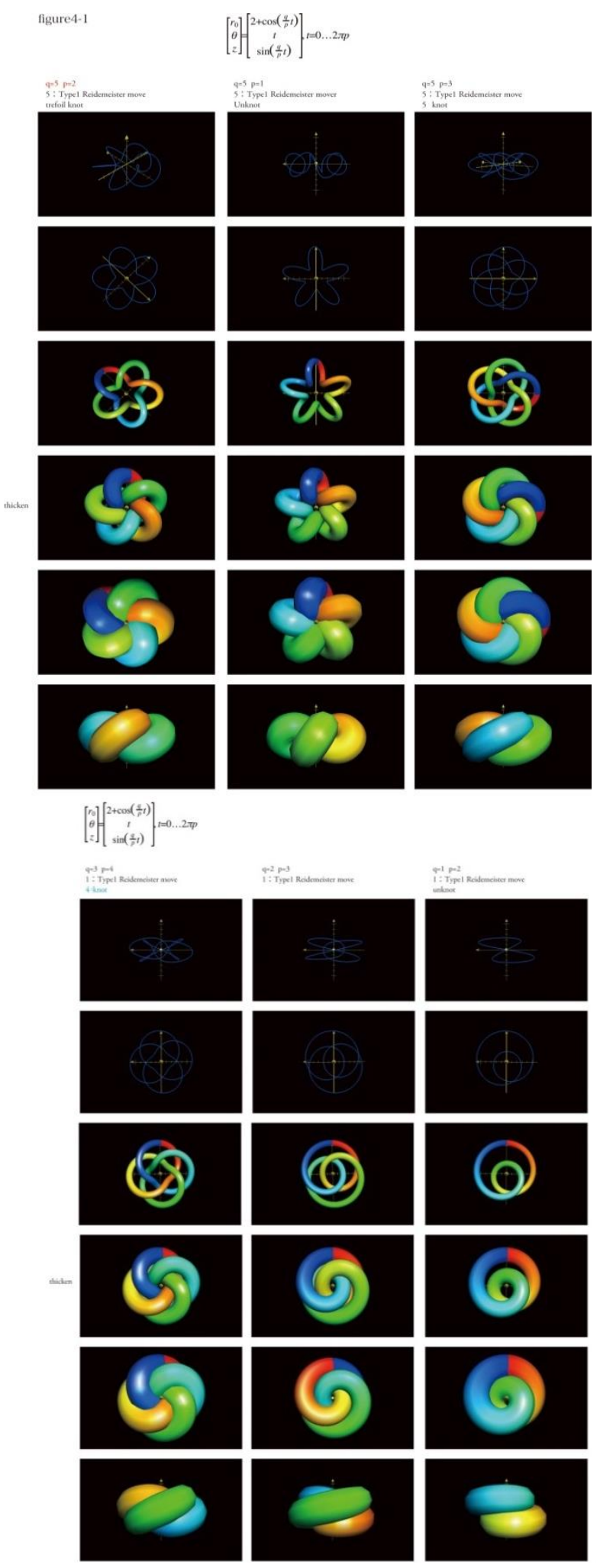

Fig. 4. The transformation and regeneration of a doughnut-shaped torus of a three-dimensional open manifolds normally perceived as " 1 "
The "separation" boundary problem may be perceived using substances that function as the smallest grinders during the cell division of an organism. These substances may be related to the 17 types of metals (halogen metals) subjected to epitope induction, have dielectric polarization properties and they may be able to transform into shapes such as Kepler manifolds. It is considered that they form cells while in motion. In other words, they could be considered to have the dielectric polarization characteristics of the 17 types of metals, which is the result of the reactions to epitope inductions. The initial setting of the continuum hypothesis has been examined only by the concept, but some ambiguity remains therein. It is necessary to examine the definition of the number before proving the consistency of the number, and in particular to examine why it has reached the point where it is recognized in that manner.

That decimal is, in a compressed description of the calculation in a two-dimensional, slightly deviated from the phenomenon of a three-dimensional space of reality.

Described above

\section{1) biological model \\ 2) machine model \\ 3) conceptual model \\ Respectively \\ 1) 4 or more dimensions model \\ 2) two-dimensional model \\ 3) 3-dimensional model \\ That is the meaning.}

These extra dimensions (intermediate dimension), how to traffic to no contradiction, is a Exponentiation symmetry can be calculated the coating space.

Numbers can be considered to be products of light perception by organisms, and their true nature is that they move subtly depending on the dimensions of their notation. In general, they are a group with the Painlevé property.

In other words, the types of equations described here exist to describe independent organisms, making folds and lowering dimensions for clean convergence when induction has completed. To put it differently, the description of life starts from the symmetry of cell division. When it is assumed that two-point discrimination cognition has established current mathematical logic, it was thought that some condition or other with epitope induction is carrying out the recognition of the boundary problems thereof, but it is precisely this "some condition or other" that may be a Painlevé property that has Exponentiation symmetry.

\section{REFERENCES}

[1] Barbara McClintock "The Significance of Responses of the Genome to Challenge", Science 1984 vol226 November Copyright $\odot 1984$ by the Nobel Foundation

[2] Bruce Alberts, Julian Lewis, Martin Raff, "Molecular Biology of the Cell” , Newton Press Co.,Ltd. 2010 ISBN-10: 4315518670

[3] Christopher Bishop "Pattern Recognition and Machine Learning" , Maruzen Co.,Ltd. 2009.

[4] J.H.Conway D.A.Smith "On uaternions and ctonions" , A K Peters,Lte 2003 ISBN 1-56881-134-9 
[5] J.P.Cohen “ Continuum hypothesis” , TokyoTosho Co.,Ltd. 1972 ISBN 4-489-00339-0

[6] Katsusada Morita “ On uatrenions and ctonions and dirac equation" , Nippon Hyoron Sha Co.,Ltd 2011 ISBN 978-4-535-78676-9

[7] Kenji Fukaya "Electromagnetic fields and the vector analysis" , Iwanami Shoten Publishers 2006 ISBN 4-00-006883-0

[8] Linus Pauling "A Molecular Theory of General Anesthesia”, Science 7 July 1961 vol 134 November 3471
[9] Masatoshi Noumi "Painleve equation", Asakura Publishing Co.,Ltd. 2000 ISBN 978-4-254-11554-3

[10] Tosaka, Nobuyoshi "Solution and application of differential equation" , University of Tokyo Press 2010 ISBN 978-4-13-062913-3

[11] Yukio Matsumoto "Basis of the manifold" , University of Tokyo Press 1988 ISBN 4-13-062103-3

[12] William Poundstone“ The Recursive Universe” , Nippon Hyoron Sha Co.,Ltd , 1990 